\title{
Confocal Fluorescence Imaging Enables Noninvasive Quantitative Assess- ment of Host Cell Populations In Vivo Following Photodynamic Therapy
}

\author{
Soumya Mitra, Oleg Mironov, and Thomas H. Foster ${ }^{\bowtie}$ \\ Department of Imaging Sciences, University of Rochester Medical Center, Rochester, NY 14642, USA.
}

Corresponding author: Soumya Mitra, University of Rochester, Department of Imaging Sciences, 601 Elmwood Avenue, Box 648, Rochester, NY 14642, USA. Email: soumya.mitra@rochester.edu, Phone: 585-275-3645.

(C) Ivyspring International Publisher. This is an open-access article distributed under the terms of the Creative Commons License (http://creativecommons.org/ licenses/by-nc-nd/3.0/). Reproduction is permitted for personal, noncommercial use, provided that the article is in whole, unmodified, and properly cited.

Received: 2012.03.20; Accepted: 2012.06.14; Published: 2012.09.14

\begin{abstract}
We report the use of optical imaging strategies to noninvasively examine photosensitizer distribution and physiological and host responses to 2-[I-hexyloxyethyl]-2 devinyl pyropheophorbide-a (HPPH)-mediated photodynamic therapy (PDT) of EMT6 tumors established in the ears of BALB/c mice. $24 \mathrm{~h}$ following intravenous (IV) administration of I $\mu \mathrm{mol} \mathrm{\textrm { } \mathrm { g } ^ { - 1 }}$ $\mathrm{HPPH}$, wide-field fluorescence imaging reveals tumor selectivity with an approximately 2-3-fold differential between tumor and adjacent normal tissue. Confocal microscopy demonstrates a relatively homogeneous intratumor HPPH distribution. Labeling of host cells using fluorophore-conjugated antibodies allowed the visualization of $\mathrm{GrI}^{+} / \mathrm{CDI} \mathrm{Ib}^{+}$leukocytes and major histocompatibility complex class II (MHC-II) ${ }^{+}$cells in vivo. Imaging of the treated site at different time-points following irradiation shows significant and rapid increases in $\mathrm{GrI}^{+}$ cells in response to therapy. The maximum accumulation of $\mathrm{Grl}^{+}$cells is found at $24 \mathrm{~h}$ post-irradiation, followed by a decrease at the $48 \mathrm{~h}$ time-point. Using IV-injected FITC-conjugated dextran as a fluorescent perfusion marker, we imaged tissue perfusion at different times post-irradiation and found that the reduced $\mathrm{Grl}^{+}$cell density at $48 \mathrm{~h}$ correlated strongly with functional damage to the vasculature as reported via decreased perfusion status. Dual color confocal imaging experiments demonstrates that about $90 \%$ of the anti-Grl cell population co-localized with anti-CDIIb labeling, thus indicating that majority of the Grl-labeled cells were neutrophils. At $24 \mathrm{~h}$ post-PDT, an approximately 2-fold increase in $\mathrm{MHC}-\mathrm{Il}+$ cells relative to untreated control is also observed. Co-localization analysis reveals an increase in the fraction of $\mathrm{GrI}^{+}$cells expressing MHC-II, suggesting that HPPH-PDT is stimulating neutrophils to express an antigen-presenting phenotype.
\end{abstract}

Key words: Photodynamic therapy, HPPH, In vivo imaging, Intratumor drug distribution, Confocal fluorescence microscopy, Immune cell imaging.

\section{Introduction}

Photodynamic therapy (PDT) continues to gain clinical acceptance worldwide as a minimally invasive treatment for neoplastic disease [1-3]. In the United States, FDA approval has been given to Porfimer sodium (Photofrin), a first-generation photosensitizer, for the treatment of Barrett's esophagus with high grade dysplasia, obstructing esophageal carcinoma and early and obstructing tracheobronchial carcinoma. Drawbacks posed by Photofrin include prolonged skin photosensitivity and limited tumor selectivity in patients [2]. In an effort to address this challenge, several next generation photosensitizers are being evaluated. Among them, 2-[1-hexyloxyethyl]-2 devinyl pyropheophorbide-a (HPPH) has emerged as a 
promising candidate. HPPH-PDT has demonstrated excellent safety and efficacy in the treatment of Barrett's esophagus, oral cavity cancers, and early and late stage esophageal and lung cancers [4-6]. Further, HPPH has demonstrated minimal skin photosensitization in preclinical and clinical studies [7].

As drug biodistribution is an important component in determining treatment response and selectivity, several studies have evaluated pharmacokinetics of $\mathrm{HPPH}$ and its effectiveness in inducing tumor destruction with different drug-light intervals $[4,8]$. These pharmacokinetic measurements have demonstrated that the half-life of HPPH in plasma is $20-26$ $\mathrm{h}$, which may be compared to the significantly longer elimination half-life of approximately $200 \mathrm{~h}$ with Photofrin [8]. This 10-fold lower plasma half-life is the primary reason for the reduced cutaneous photosensitivity associated with HPPH. A study by Lobel et al. in a rat glioma model examined the tumor tissue selectivity for HPPH [9]. They reported a 3:1 tumor-to-normal brain tissue ratio of HPPH concentration at $24 \mathrm{~h}$ post-administration with a half-life in tumor tissue of approximately $30 \mathrm{~h}$. However, no studies have yet examined the intratumor distribution of HPPH. We have previously reported on the use of imaging techniques to evaluate the uptake and distribution of several photosensitizers in tumor tissue [10-12]. In this study we investigated the selectivity of $\mathrm{HPPH}$ in tumors and its intratumor distribution at a drug-light interval of $24 \mathrm{~h}$ using in vivo fluorescence imaging performed in tumors established in ears of mice.

Recruitment of inflammatory cells has important effects on tumor development, and the role of these cells as anti- or pro-tumor agents is a subject of continuing investigation by several groups [13]. In the context of PDT, pre-clinical studies have however demonstrated that effective long term response following therapy is achieved not only by direct oxidative damage to tumor cells and vasculature but also by multiple elements of the host response [14]. These responses, possibly triggered by PDT-induced inflammation, are characterized by increased expression of proinflammatory cytokines and adhesion molecules and rapid leukocyte infiltration into the treated tumor. A significant fraction of these infiltrating leukocytes are neutrophils $[1,15]$. de Vree et al. were one of the first groups to report the enhanced accumulation of neutrophils in peripheral blood following PDT irradiation, and the significant contribution of neutrophils was further confirmed with experiments in animal models where depletion of neutrophils using anti-Gr1 monoclonal antibodies resulted in diminished Photofrin-PDT efficacy [16, 17]. Gollnick et al. have shown that PDT with HPPH also initiates a local inflammatory response characterized by neutrophil migration into the treated tumor, albeit less pronounced than that observed with Photofrin PDT [18]. A subsequent study by Kousis et al. elucidated the role of these infiltrating neutrophils in augmenting T-cell proliferation, and thus suggested for the first time a mechanistic link between the acute inflammatory response to PDT and tumor-specific, systemic immunity [19].

Immune responses induced by PDT have thus far been examined using traditional biochemical assays, immunohistochemistry of tissue sections, or flow cytometry. However, these assays and techniques require tissue extraction and processing. To address these limitations and study inflammatory response in minimally perturbed tissue architecture, we undertook a detailed examination of kinetics of host cell infiltration evoked by PDT treatment using a recently reported in vivo optical imaging approach $[11,12]$. The technique exploits local administration of fluorophore-conjugated antibodies against cell-specific surface antigens and enables imaging in live mice the population of $\mathrm{Gr}^{+}$and $\mathrm{MHC}-\mathrm{II}^{+}$cells in $\mathrm{HPPH}$-treated tissue at different times post-treatment. Using this imaging technique, we demonstrate that a strong inflammatory response characterized with rapid and increased influx of $\mathrm{Gr}^{+}$cells is associated with a PDT dose that yields $90 \%$ tumor cures. We also observe that in response to PDT, a modest but significantly greater number of $\mathrm{Gr}^{+}$cells express major histocompatibility complex class II (MHC-II), thus suggesting a role for them as antigen presenters.

\section{Materials and Methods}

Cell culture and tumor model: Mouse mammary cancer EMT6 cells were maintained in monolayer culture in Eagle's basal medium (BME) with 10\% fetal bovine serum (complete media) at $37^{\circ} \mathrm{C}$. EMT6 tumors were initiated by injection of $5 \times 10^{5}$ EMT6 cells into the ear pinna of $4-6$ weeks old female BALB/c mice. Animals were followed daily to track tumor growth, and 7 - 10 days after implantation when the tumors reached a volume of approximately $15-25 \mathrm{~mm}^{3}$ they were used for experiments as described below.

Photosensitizer administration and light treatment: Mice bearing EMT6 ear tumors were administered $1 \mu \mathrm{mol} \mathrm{kg}-1$ of the photosensitizer $\mathrm{HPPH}$ via intravenous (IV) tail vein injection. At $24 \mathrm{~h}$ post HPPH administration, the tumors were subjected to PDT irradiation using $667 \mathrm{~nm}$ light from a diode laser (Power Technology Inc., Little Rock, AR). Light was delivered through a GRIN-lens-terminated multi- 
mode fiber (OZ Optics, Ottawa, ON, Canada), and the tumors were illuminated with a fluence of $100 \mathrm{~J} \mathrm{~cm}^{-2}$ at an irradiance of $75 \mathrm{~mW} \mathrm{~cm} \mathrm{~cm}^{-2}$. Controls included untreated ((-) drug, (-) light) animals.

Tumor-response assay: Tumor growth control was used to evaluate the therapeutic efficacy of the HHPH-PDT treatment parameters used for this study. After PDT, tumor dimensions along 3 orthogonal axes were measured daily using digital calipers. Volumes were computed assuming an approximately ellipsoidal shape with the expression, $\mathrm{V}=(4 / 3) \Pi \mathrm{r}_{1} \mathrm{r}_{2} \mathrm{r}_{3}$. Mice were removed from the study if the volume of the tumor reached twice the pre-treatment volume. Cures were defined as no evidence of palpable tumor 90 days after PDT.

In vivo imaging of photosensitizer uptake and perfusion status using stereofluorescence microscopy: In order to observe large fields of view at high magnification, imaging was performed over the ear tumor of an anesthetized mouse using a Nikon fluorescence stereomicroscope (model SMZ-1500, Nikon Instruments, Melville, NY) equipped with an Xcite illumination source (EXFO, Ontario, Canada). Prior to imaging, hair was removed from the imaged site using Nair. The stereoimaging system can acquire individual fields of view (FOV) as large as $2.7 \mathrm{~cm} \times 2.0 \mathrm{~cm}$, which is large enough to image the entire outer ear. Excitation of $\mathrm{HPPH}$ and its fluorescence collection were performed using a custom filter cube (HQ560/120x; 635 DCXR; HQ645LPm, Chroma Technology, Bellows Falls, VT). HPPH localization in the tumor was imaged at $24 \mathrm{~h}$ following administration. To visualize perfusion status, $200 \mu \mathrm{L}$ of $5 \mathrm{mg}$ $\mathrm{mL}^{-1}$ fluorescein isothiocyanate (FITC)-dextran (FD2000S, Sigma-Aldrich, St. Louis, MO) was injected IV via tail vein. Tumor perfusion was examined at different time-points ranging from pre-irradiation to $48 \mathrm{~h}$ following PDT. FITC fluorescence was excited and detected using the ENDOW GFP bandpass filter cube (Chroma). High-resolution images of $1390 \times 1040$ pixels were captured and digitized by a Photometrics 12-bit monochrome CCD camera (CoolSNAPHQ, Roper Scientific, Inc., Trenton, NJ).

In vivo confocal imaging of intratumor photosensitizer distribution, host responses and perfusion status: In vivo imaging of photosensitizer distribution, host responses or perfusion status in tissue on a microscopic scale was performed using a custom laser scanning fluorescence confocal microscope described previously [20]. Briefly, anesthetized mice were placed in a supine position with the ear tumors facing the top of a coverslip mounted on the stage of the inverted microscope. The fluorophores AlexaFluor647 and Allophycocyanin were excited with a 639 $\mathrm{nm}$ diode laser and detected using a $647 \mathrm{~nm}$ long pass filter (Semrock, Rochester, NY). AlexaFluor488 and FITC were excited at $488 \mathrm{~nm}$ from an argon ion laser and detected using a combination of $500 \mathrm{~nm}$ long pass and 515/30 bandpass filters (Chroma). The combination of a $100 \mu \mathrm{m}$ diameter pinhole and a 10x, $0.45 \mathrm{NA}$ objective gave a $6 \mu \mathrm{m}$ optical section thickness, and the images were acquired at 16 bits with a lateral resolution of $1 \mu \mathrm{m}$ per pixel.

For immunofluorescence imaging, in vivo antibodies were purchased directly as fluorescent conjugates. We labeled leukocytes using intradermal (ID) administration of $3 \mu \mathrm{g}$ of anti-mouse Gr1 and CD11b antibodies (Gr1: clone RB6-8C5; CD11b: clone M1/70, Biolegend, San Diego, CA) and imaged their population at different times post irradiation. Major histocompatibility complex class II cells were labeled with antibodies against MHC-II (1.5 $\mu \mathrm{g}$; Clone M5/114.15.2, Biolegend). AlexaFluor647- or AlexaFluor488-conjugated anti-CD31 antibody (clone MEC13.3, Biolegend) was administered ID in the mouse ear to label the vessels. The ID injection volumes of antibodies were approximately $20 \mu \mathrm{L}$ and were administered $3 \mathrm{~h}$ prior to imaging to allow for clearance of unbound label.

We investigated the microscopic intratumor distribution of $\mathrm{HPPH} 24 \mathrm{~h}$ following administration. $\mathrm{HPPH}$ was excited with $514 \mathrm{~nm}$ from an argon ion laser, and its emission was detected using a combination of $525 \mathrm{~nm}$ and $647 \mathrm{~nm}$ long pass filters (Chroma). Vessel perfusion status was imaged in control tissue pre-irradiation and at 24 and $48 \mathrm{~h}$ following HPPH-PDT. For any given time-point examined, perfusion in $\mathrm{CD} 1^{+}$vasculature was imaged as early as 5 min post-injection of FITC-dextran.

Image analysis: The images were analyzed using Image $1.43 \mathrm{u}$, a public domain image processing software distributed by the NIH (http://rsbweb.nih.gov/ij/). All images had fields of view of $800 \mu \mathrm{m} \times 800 \mu \mathrm{m}$. To obtain cell density in an imaged field, grayscale images of antibody fluorescence were processed to mask $\mathrm{Gr} 1^{+}$cells. Next, the images were thresholded into a binary format. A watershedding algorithm was applied, and the resulting particles were screened for size using the Analyze tool in ImageJ. Size screening was based on the assumption that $\mathrm{Gr}^{+}$cells were at least $8 \mu \mathrm{m}$ in diameter and therefore had a cross sectional area of approximately $50 \mu \mathrm{m}^{2}$. The results were analyzed using $\mathrm{R}$ version 2.12.0 ( $\mathrm{R}$ foundation for Statistical Computing; http://www.r-project.org/). Cell counts at different time intervals were compared using an unpaired 2-tailed t-test without assuming equal variances.

Co-localization analysis: The extent of 
co-localization between the fluorescence of $\mathrm{Gr}^{+}$and MHC-II' pixels or between $\mathrm{Gr}^{+}$and $\mathrm{CD}^{+} 1 \mathrm{~b}^{+}$pixels was determined using the Intensity Correlation Analysis tool in ImageJ, a statistical approach based on a cross-correlation analysis introduced by Manders et al. [21]. This analysis yielded coefficients that quantified the co-localized fraction of the signal in the two channels above a specified intensity threshold. The details of the analysis have been reported in a recent study [22].

\section{Results and Discussion}

The Kaplan-Meier curves of Figure 1 demonstrate the tumor response to a fluence of $100 \mathrm{~J} \mathrm{~cm}^{-2}$ delivered at an irradiance of $75 \mathrm{~mW} \mathrm{~cm}^{-2}$. Among the 10 mice treated with this PDT dose, $9(90 \%)$ were cured as defined by no evidence of tumor 90 days after irradiation. Untreated controls $((-)$ light, (-) drug) displayed a median tumor doubling time of 6 days $(n=4)$.

In vivo, whole-mouse fluorescence imaging using the stereofluorescence microscope showed 2-3-fold tumor-to-normal skin selectivity (Figure 2(a)) at $24 \mathrm{~h}$ following IV administration of HPPH. We recently reported on the intratumor distribution of the sensitizer, NPe6, wherein we used the antibody labeling technique to label $\mathrm{CD} 31^{+}$tumor vasculature and imaged the spatial distribution and temporal kinetics of NPe6 with respect to vessels following systemic administration [11]. Adopting the same approach, we examined the microscopic pattern of intratumor HPPH distribution using confocal fluorescence imaging.

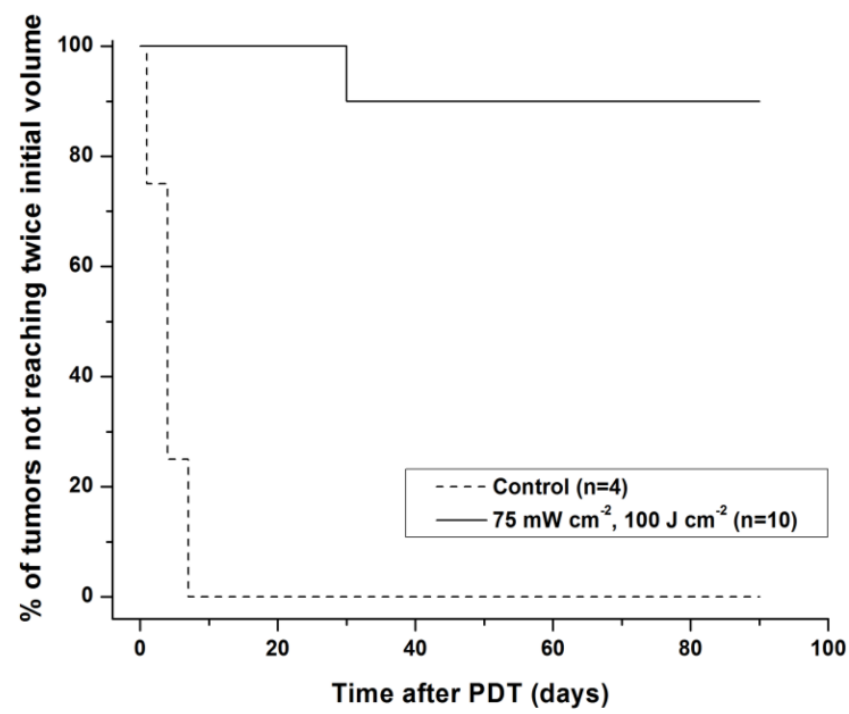

Figure I: Kaplan-Meier curves of tumor responses to HPPH-PDT. Laser irradiation at $667 \mathrm{~nm}$ was performed at an irradiance of $75 \mathrm{~mW} \mathrm{~cm}^{-2}$ for a fluence of $100 \mathrm{~J} \mathrm{~cm}^{-2}$ at $24 \mathrm{~h}$ after intravenous administration of $\mathrm{I} \mu \mathrm{mol} \mathrm{kg}$ $\mathrm{HPPH}$.
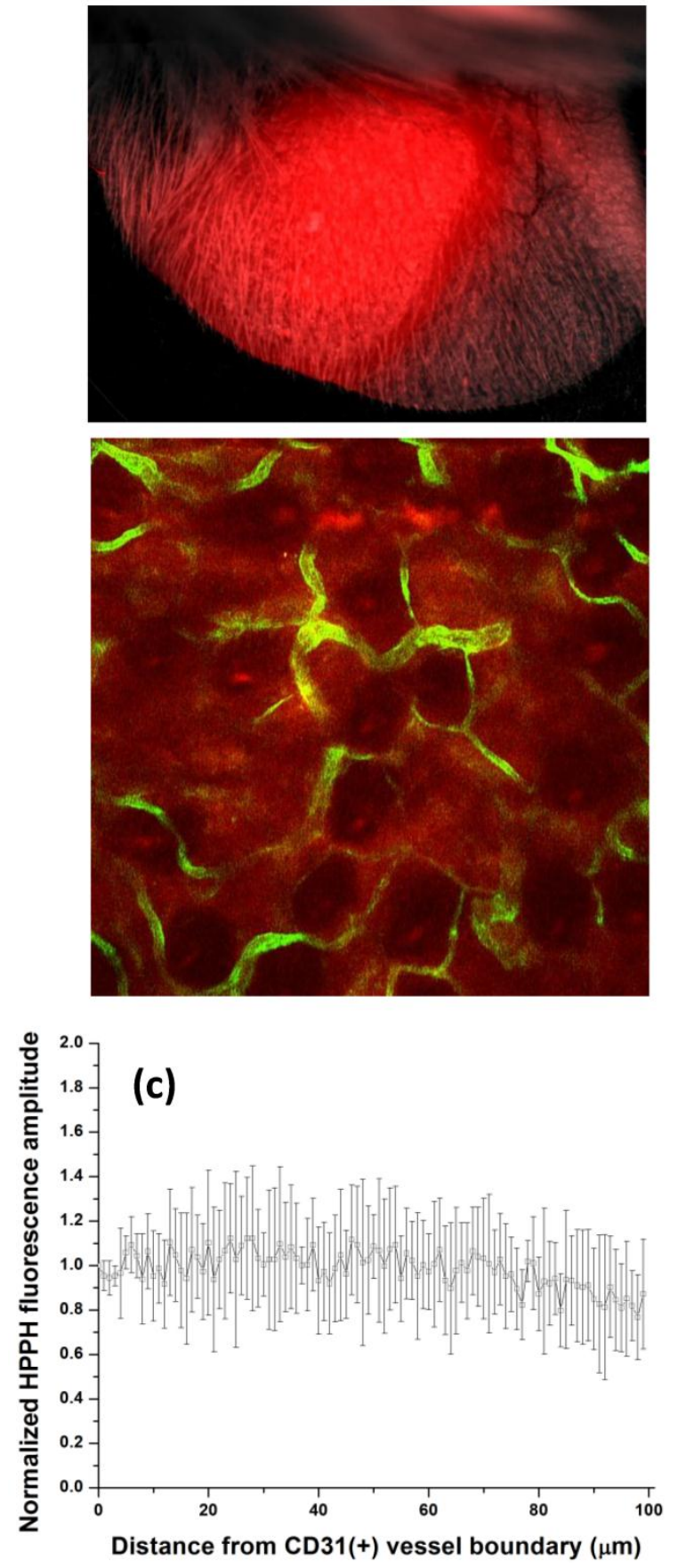

Figure 2: (a) Stereofluorescence image of an EMT6 tumor in the ear of a $\mathrm{BALB} / \mathrm{c}$ mouse, acquired at $24 \mathrm{~h}$ post IV administration of I $\mu \mathrm{mol} \mathrm{kg}^{-1}$ HPPH. This representative image is a superposition of a bright field and HPPH fluorescence and highlights the selective uptake of HPPH in the tumor tissue. The HPPH fluorescence intensity in the tumor is approximately 2-3-fold higher than the adjacent normal tissue. (b) In vivo confocal image of intratumor HPPH distribution imaged at a depth of $100 \mu \mathrm{m}$ into a mouse ear. HPPH fluorescence is in red, and $\mathrm{CD}_{3} \mathrm{I}^{+}$vessels labeled by Alexa488-conjugated antibody are in green. (c) HPPH fluorescence as a function of distance from $\mathrm{CD}_{3} \mathrm{I}^{+}$vessels, normalized to the fluorescence amplitude at the vessel boundary. The data points are an average of at least four independent measurements, and the error bars represent standard deviations. 
Figure 2(b) shows a fluorescence image of $\mathrm{HPPH}$ distribution (red) with respect to anti-CD31 labeled vasculature (green) at a depth in tissue of $100 \mu \mathrm{m}$. The image was acquired $24 \mathrm{~h}$ following IV injection of 1 $\mu \mathrm{mol} \mathrm{kg}^{-1} \mathrm{HPPH}$. As illustrated, at this time point $\mathrm{HPPH}$ has partitioned from the vasculature into the adjacent parenchyma relatively uniformly. Figure 2(c) shows analysis of HPPH distribution from fields of view from four independent experiments. The mean $\mathrm{HPPH}$ fluorescence amplitude is plotted as a function of distance from a vessel wall. Even at a distance of $100 \mu \mathrm{m}$ the HPPH fluorescence does not drop below $30 \%$ of the intensity at the vessel boundary.

PDT induces a local inflammatory response that is characterized by leukocyte infiltration, with a significant fraction of these infiltrating cells being neutrophils [23]. To examine the extent of this response to HPPH-PDT using treatment parameters that resulted in $90 \%$ tumor cures, we imaged the influx of $\mathrm{Gr}^{+}$cells in vivo into the treated site at 5, 24 and $48 \mathrm{~h}$ post-irradiation. Figures $3(\mathrm{a})$ and (b) illustrate the fluorophore-labeled infiltrating $\mathrm{Gr}^{+}$cells imaged in an untreated control and PDT-treated ear, respectively. The image shown in figure $3(\mathrm{c})$ is a magnified
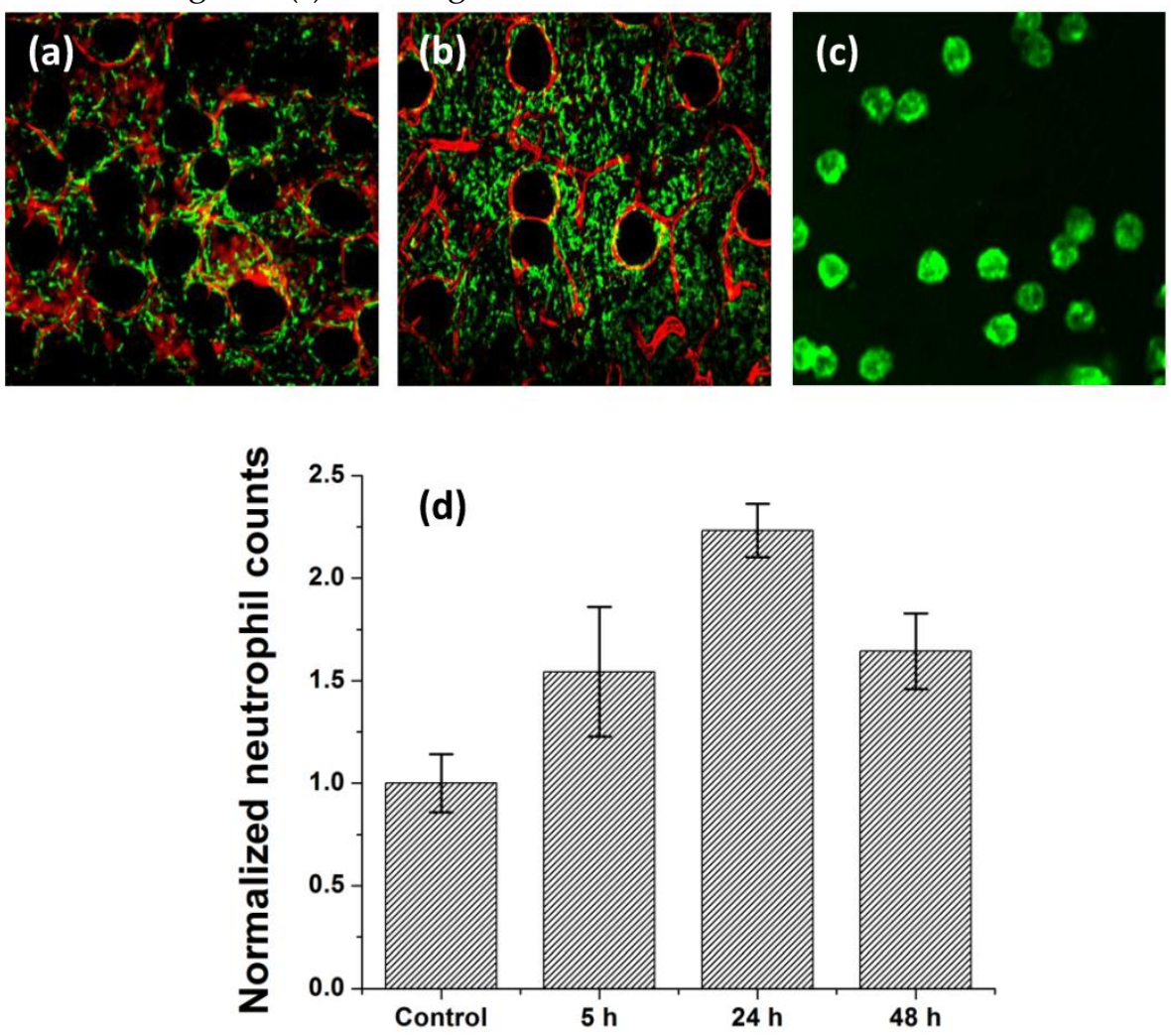

Figure 3: In vivo confocal images of Alexa488-conjugated anti-Gr ${ }^{+}$cells (green) and Alexa647-conjugated anti-CD3I vessels (red) in the tumor-bearing ears of BALB/c mice.. (a) Control ear that received HPPH but was not irradiated, and (b) ear that was PDT-treated. The FOV in these images is $800 \mu \mathrm{m} x$ $800 \mu \mathrm{m}$. The image in (c) is an expanded view of an anti-Grl imaged field to illustrate the high contrast staining of host cells. (d) Mean normalized neutrophil counts in control and PDT-treated sites at time-points of 5, 24 and 48 hours following irradiation. $24 \mathrm{~h}$ post-PDT, the treatment induces approximately 2.5 -fold higher neutrophil accumulation in the irradiated tissue. The neutrophil counts at $24 \mathrm{~h}$ are significantly different from those at control, $5 \mathrm{~h}$ and 48 h time-points $(P<0.05)$. view of an imaged field and demonstrates that confocal imaging can resolve the antibody-labeled $\mathrm{Gr}^{+}$ cells at an individual cell level. The data summarized in figure $3(\mathrm{~d})$ from multiple independent measurements show an approximately 2.5 -fold enhanced $\mathrm{Gr}^{+}$ cell infiltration in the treated site $24 \mathrm{~h}$ post-irradiation smaller but significant increase in $\mathrm{Gr}^{+}$cell accumulation as early as $5 \mathrm{~h}$ following treatment. At $48 \mathrm{~h}$ post-irradiation, the $\mathrm{Gr}^{+}$cell counts decrease and are not significantly different from those at the $5 \mathrm{~h}$ time-point. This trend in $\mathrm{Gr}^{+}$cell accumulation is remarkably similar to that reported by Gollnick et al. in an elegant study that used identical HPPH-PDT treatment conditions to investigate various mediators of the inflammatory response [18]. Using flow cytometry, the authors found that HPPH-PDT induces an influx of nearly 3-fold higher neutrophils into tumors compared to untreated control at $24 \mathrm{~h}$ post-irradiation. The comparable finding between our study and that reported by Gollnick et al. serves as a measure of validation for the in vivo imaging strategy as an assay for host cell populations. compared to untreated control. We also observe a 
Perfused tumor blood vessels have important functions as the source of local tumor oxygenation and as a route for host cell trafficking [24]. With this motivation, bulk tumor perfusion in vivo was imaged with the stereofluorescence microscope, and microscopic imaging of small numbers of individual vessels was performed using the confocal microscope. Perfusion in live mice was visualized at pre-, $24 \mathrm{~h}$, and $48 \mathrm{~h}$ post-irradiation using IV-injected FITC-conjugated high molecular weight dextran as an optical perfusion marker. Figures $4(\mathrm{a}-\mathrm{c})$ are representative stereofluorescence images of perfusion status in an EMT6 ear tumor in the same mouse, followed up to $48 \mathrm{~h}$ post-PDT. As illustrated in figures 4(a) and 4(b), there was no detectable difference in perfusion status between the control and treated tumor at $24 \mathrm{~h}$ post-treatment. However, at the $48 \mathrm{~h}$ time-point a severe perfusion deficit is observed (figure 4(c)). Figures 4(d-f) are confocal images of microscopic patterns of perfusion level (green) in CD31+ labeled vessels (red). Consistent with the stereofluorescence images, we find that relative to the highly perfused vessels observed in control tissue, most of the CD31-positive vasculature in the treated region at $48 \mathrm{~h}$ post-irradiation exhibits an absence or low levels of the perfusion marker. This observation of perfusion loss at the $48 \mathrm{~h}$ correlates positively with the reduced $\mathrm{Gr}^{+}$population density at the same time-point. It is well established that vascular damage may be mediated by neutrophil secretion products such as chemokines, heparin-binding protein and arachidionic acid $[25,26]$. Therefore, the reduced perfusion status at 48 $h$ post-PDT is likely triggered by the large accumulation of neutrophils in the tumor tissue. This mode of vascular response is in contrast to the vascular shutdown induced by Visudyne-PDT, where direct damage to ECs leads to rapid loss of vascular barrier function [27]. Further, recent evidence has shown that neutrophils release proteolytic enzymes like MMP-9 upon activation [28]. Therefore, degradation of the extracellular matrix and damage to blood vessels following HPPH-PDT may also be mediated in part by the release of MMPs from the accumulated neutrophil population.

Gr1 is expressed on neutrophils, macrophages, and plasmacytoid dendritic cells [29, 30]. Therefore, the results reported above could potentially reflect cell populations other than neutrophils. In flow cytometry, identification of neutrophils includes positive staining for Gr1 and CD11b and negative staining for F4/80 [19]. We attempted to label in vivo with antibody against F4/80 but observed that commercially available fluorophore-conjugated anti-F4/80 antibodies resulted in unacceptably poor staining.
Other investigators have reported similar issues with anti-F4/ 80 for ex-vivo tissue staining protocols [31]. In the absence of a reliable F4/80 marker and in an effort to further scrutinize the $\mathrm{Gr} 1^{+}$cells in the images, we therefore performed two-color imaging experiments in several control and treated ears using an antibody cocktail that contained anti-Gr1 and anti-CD11b conjugated to AlexaFluor647 and AlexaFluor488, respectively. Figures 5(a) and 5(b) are representative in vivo images from identical fields of view $24 \mathrm{~h}$ post-PDT that are comprised exclusively of anti-CD11b or anti-Gr1 fluorescence when excited by 488 or $639 \mathrm{~nm}$ light, respectively. Figure 5(c) is a merged image of the two channels and displays strong overlap between the two channels in pixels exhibiting yellow / orange color. The high degree of overlap between pixels that display anti-CD11b and anti-Gr1 labeling is confirmed by co-localization analysis, which yields Manders coefficients of approximately $90 \%$. These coefficients in the treated tissue at $24 \mathrm{~h}$ and $48 \mathrm{~h}$ post-HPPH-PDT were not different from those measured in untreated controls.

We have recently described imaging of ${\mathrm{MHC}-\mathrm{II}^{+}}^{+}$ cells in normal and tumor tissue accomplished using fluorescence labeling in vivo [32]. MHC class II proteins are present on the surface of antigen presenting cells (APCs), majority of which are macrophages and dendritic cells. It is well established that these cells internalize antigens and display a fragment of the antigen, bound to a MHC-II molecule which is recognized by T-cells, leading to their activation and initiation of specific immunity. We therefore imaged the MHC-II+ cell population in untreated control and $24 \mathrm{~h}$ post-PDT treated tissue. Figures $6(\mathrm{a})$ and $6(\mathrm{~b})$ illus-

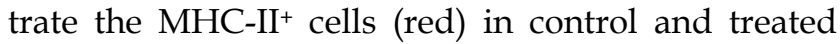
tissue, respectively. The distribution of $\mathrm{MHC}^{-\mathrm{II}^{+}} \mathrm{ob}-$ served at a depth of about $70 \mu \mathrm{m}$ is demonstrated in these representative images with respect to $\mathrm{CD} 31^{+}$ vessels (green). In contrast to the 2-3-fold PDT-induced increase in infiltration of $\mathrm{Gr}^{+}$cells (figure 3), our analysis shows a modest but significant increase in the population of $\mathrm{MHC}^{-} \mathrm{II}^{+}$cells at the treated site, with cell counts approximately 50\% higher relative to control (figure 6(c)). It is possible that infiltrating macrophages and dendritic cells contribute to this modest increase in $\mathrm{MHC}^{-\mathrm{II}^{+}}$cell counts. However, recent studies have also suggested that neutrophils under stimulation can present MHC-II on their surface and thus function as an APC [33, 34]. Motivated by these findings, we examined if HHPH-PDT can induce Gr1+ cells to present MHC-II. To test whether the fraction of $\mathrm{Gr}^{+}$cells expressing MHC-II changes in response to therapy, we imaged $\mathrm{Gr}^{+}$and $\mathrm{MHC}-\mathrm{II}^{+}$cells in the same ear following 
administration of an antibody cocktail that contained anti-Gr1 and anti-MHC-II conjugated to AlexaFluor488 and Allophycocyanin, respectively. Figures 7(a) and $7(\mathrm{~b})$ illustrate representative in vivo confocal images of anti-Gr1 ${ }^{+}$cells (green) and anti-MHC-II ${ }^{+}$ cells (red) pre- and $24 \mathrm{~h}$ post-irradiation, respectively. Co-localization analysis of these images indicates that in control tissue roughly $21 \%$ of $\mathrm{Gr}^{+}$pixels have corresponding $\mathrm{MHC}-\mathrm{II}^{+}$pixels, while in treated sites the fraction increases to approximately $28 \%$ and $36 \%$ at $5 \mathrm{~h}$ and $24 \mathrm{~h}$ post-irradiation, respectively (figure 7(c)). This suggests that PDT is inducing $\mathrm{Gr} 1^{+}$cells to express an antigen-presenting phenotype. Similar results have been reported by Sun et al., who observed expression of MHC class II on F4/80-/Gr1 ${ }^{+}$ cells that had infiltrated Photofrin-PDT-treated SCCVII tumors [23]. These results are also consistent with the observations of Kousis et al., who examined in detail the role of $\mathrm{F} 4 / 80^{-} / \mathrm{CD} 11 \mathrm{~b}^{+} / \mathrm{Gr}^{+}$neutrophils in the stimulation of adaptive immune response [19]. The authors reported that PDT-induced inflammation enables neutrophils to access tumor draining lymph nodes and directly play a role in the enhancement of T-cell activation and proliferation. Our results therefore support the contention that neutrophils may play an important role in stimulating T-cell proliferation in an MHC-II dependent manner.

In conclusion, this study takes advantage of recently established optical imaging strategies to perform an in vivo examination of determinants, ranging from drug distribution to inflammatory response, that are associated with HPPH-PDT efficacy. We present evidence that PDT conditions that yield long term tumor control elicit a strong inflammatory response characterized by influx of $\mathrm{Gr}^{+}$cells. The findings suggest a role of these $\mathrm{Gr}^{+}$cells as mediators of HPPH-PDT-induced vascular damage and as effectors of adaptive immune response through their release of several secretion products and the expression of an antigen-presenting phenotype, respectively. Our work also highlights certain limitations of optical imaging, at least as implemented here. Flow cytometry offers distinct advantages in identifying cell types using multiple markers because of its ability to label intracellular proteins, its access to angle-resolved scatter, and because contemporary flow systems utilize spectral compensation algorithms and multivariate analysis techniques that are more sophisticated than those available in our optical imaging system and others with which we are familiar.
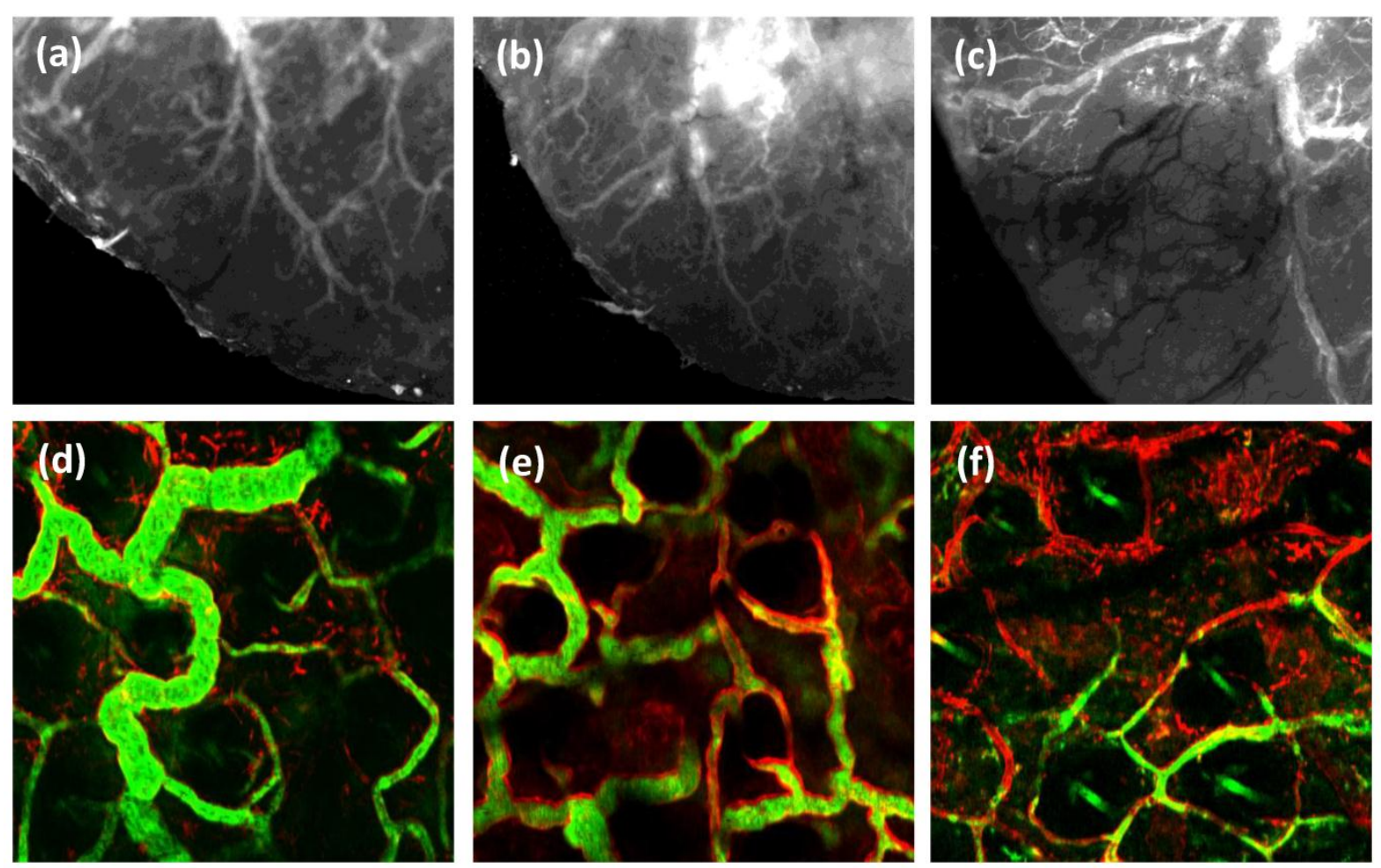

Figure 4: (a-c) Series of stereofluorescence images illustrating vessel perfusion status up to $48 \mathrm{~h}$ in an individual ear tumor treated with $100 \mathrm{~J} \mathrm{~cm}^{-2}$. (a) Untreated control, (b) $24 \mathrm{~h}$ post HPPH-PDT and (c) $48 \mathrm{~h}$ post-irradiation. These images were acquired from the same mouse tumor for 3 consecutive days. The dark vascular morphology in (c) represents tumor vessels that have lost their perfusion status. The FOV in these images is $3.6 \mathrm{~mm} \times 3.2 \mathrm{~mm}$. ( $\mathrm{d}-\mathrm{f}$ ) Representative in vivo confocal fluorescence images of perfusion (green) in CD3 $\mathrm{I}^{+}$vessels (red) in (d) untreated control, (e) $24 \mathrm{~h}$ and (f) $48 \mathrm{~h}$ post HPPH-PDT irradiation. The FOV in these images is $500 \mu \mathrm{m} \times 500 \mu \mathrm{m}$. 

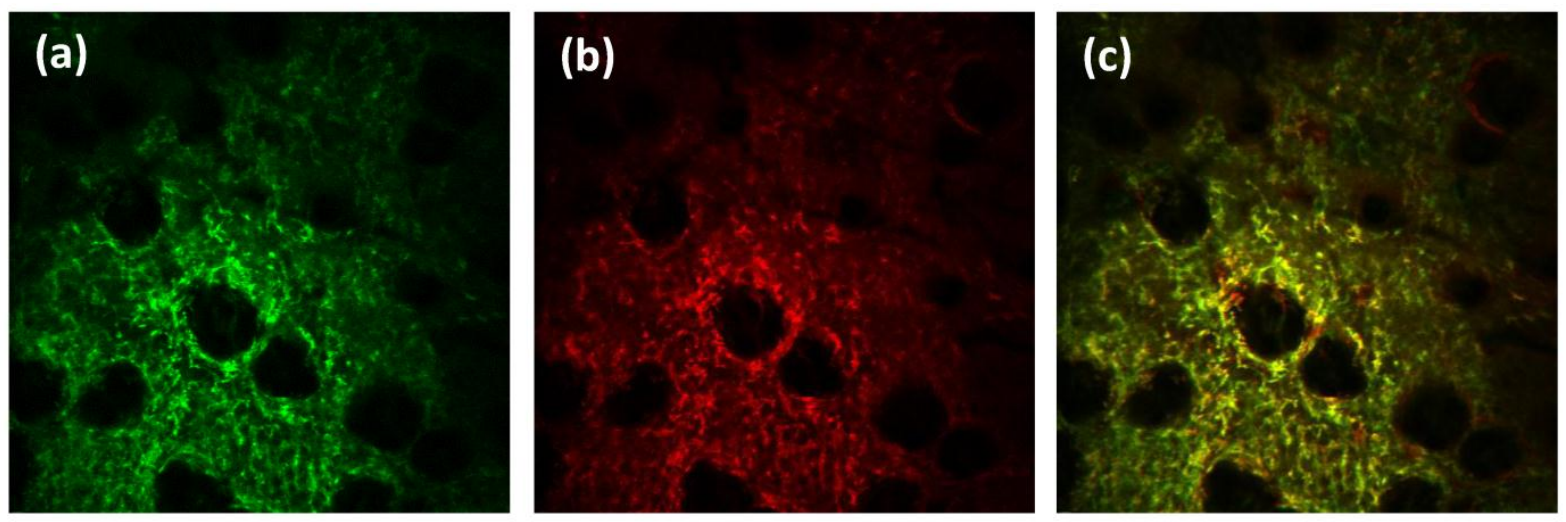

Figure 5: In vivo confocal fluorescence images of (a) $\mathrm{CDI} \mathrm{Ib}^{+}$and (b) $\mathrm{Grl}^{+}$cells in the same field of view $24 \mathrm{~h}$ post-PDT. (c) Merged image showing the extent of overlap between cells expressing CDI lb and Grl on the surface. The FOV in the images is $800 \mu \mathrm{m} \times 800 \mu \mathrm{m}$.
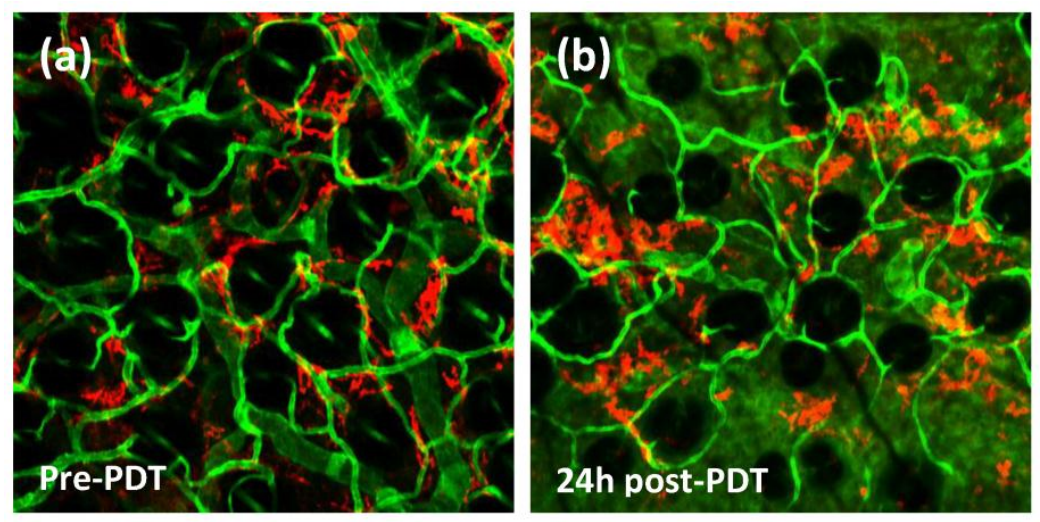

(c)

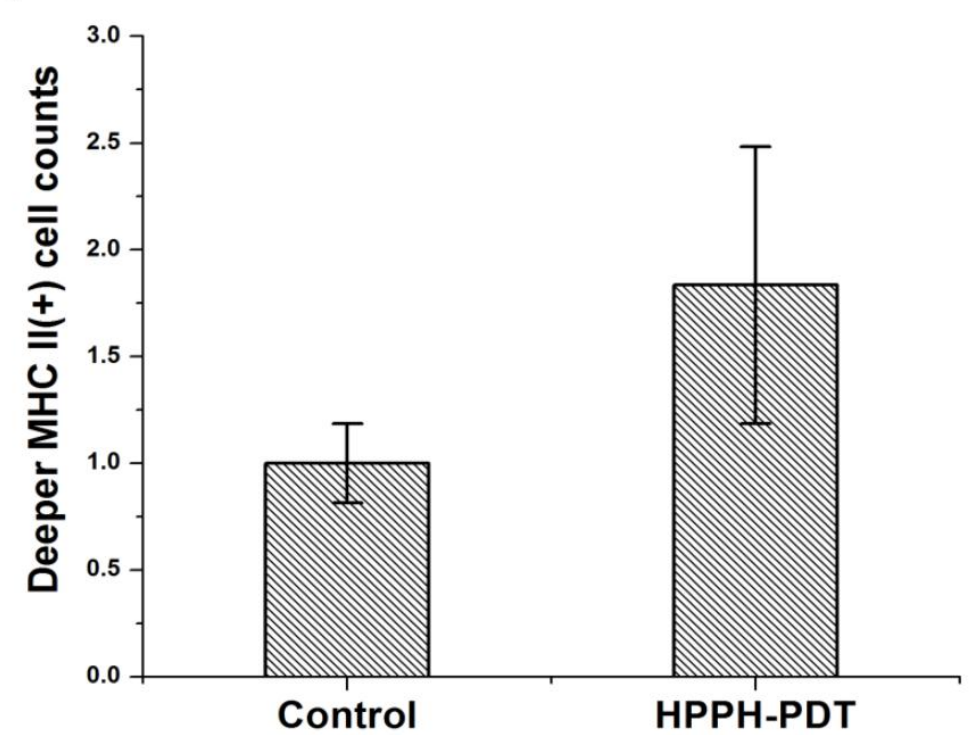

Figure 6: In vivo confocal images of MHC-II+ cells (red) and CD3I+ vessels (green). These images were acquired at depths of $70 \mu \mathrm{m}$ from the tissue surface. (a) Control ear that received HPPH but was not irradiated; (b) PDT-treated site imaged $24 \mathrm{~h}$ following irradiation. The field of view in the images is $800 \mu \mathrm{m}$ $\times 800 \mu \mathrm{m}$. (c) Mean normalized MHC- $\mathrm{II}^{+}$cell counts in untreated control and PDT-treated tissue at $24 \mathrm{~h}$ following irradiation. MHC- $\mathrm{II}^{+}$cell population is approximately 1.5 fold higher in PDT-treated vs. control tissue, and the increase is significantly different at the P=0.05 level. 

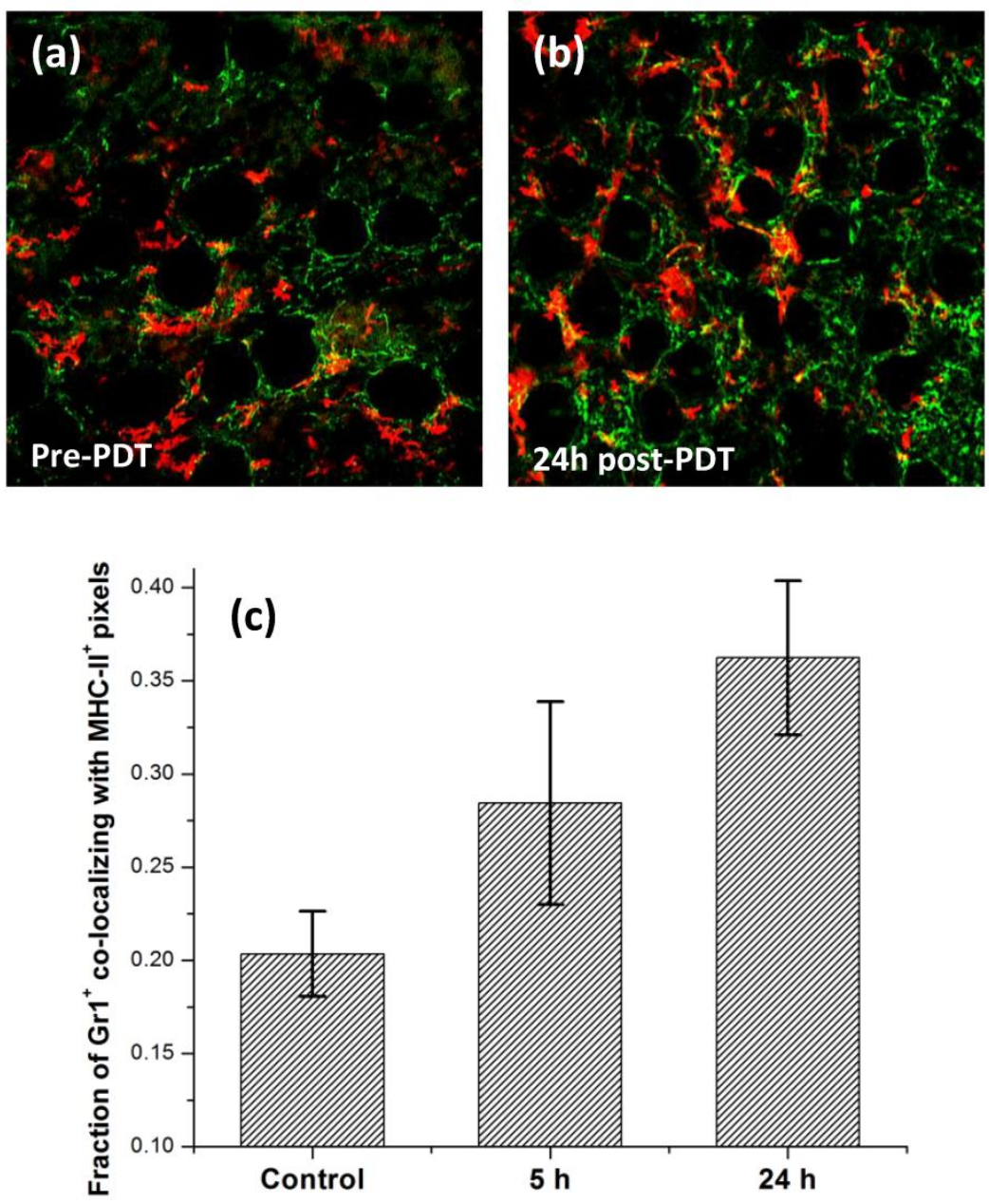

Figure 7: In vivo confocal images of Alexa488-conjugated anti-GrI ${ }^{+}$cells (green) and APC-conjugated anti-MHC-II' cells (red) in the same field of view (a) pre- and (b) $24 \mathrm{~h}$ post-irradiation. These images were analyzed to evaluate the extent of host cells that co-expressed $\mathrm{Grl}$ and MHC-II receptors on their cell surface. The FOV in the images is $800 \mu \mathrm{m} \times 800 \mu \mathrm{m}$. (c) Mean fraction of $\mathrm{GrI}^{+}$pixels co-localizing with MHC- $\mathrm{II}^{+}$pixels evaluated from at least 3 independent experiments for control, $5 \mathrm{~h}$ and $24 \mathrm{~h}$ post-irradiation. Error bars represent standard deviation. The fractions at $5 \mathrm{~h}$ vs. control and $24 \mathrm{~h}$ vs. control are significantly different at the $\mathrm{P}=0.05$ and $\mathrm{P}=0.0 \mathrm{I}$ levels, respectively.

\section{Acknowledgements}

This work was supported by National Institutes of Health grants CA68409 and P01CA55791 awarded by the National Cancer Institute. The authors thank Dr. Ravindra Pandey for generously providing HPPH. The authors thank Benjamin Giesselman for his help with the tumor response measurements.

\section{Competing Interests}

The authors have declared that no competing interest exists.

\section{References}

1. Agostinis P, Berg K, Cengel KA, Foster TH, Girotti AW, Gollnick SO, et al. Photodynamic therapy of cancer: an update. CA Cancer J Clin. 2011; 61: 250-81.

2. Harrod-Kim P. Tumor ablation with photodynamic therapy: introduction to mechanism and clinical applications. J Vasc Interv Radiol. 2006; 17: 1441-8.
3. Brown SB, Brown EA, Walker I. The present and future role of photodynamic therapy in cancer treatment. Lancet Oncol. 2004; 5: 497-508.

4. Bellnier DA, Greco WR, Loewen GM, Nava H, Oseroff AR, Pandey RK, et al. Population pharmacokinetics of the photodynamic therapy agent 2-[1-hexyloxyethyl]-2-devinyl pyropheophorbide-a in cancer patients. Cancer Res. 2003; 63: 1806-13.

5. Nava HR, Allamaneni SS, Dougherty TJ, Cooper MT, Tan W, Wilding G, et al. Photodynamic therapy (PDT) using HPPH for the treatment of precancerous lesions associated with barrett's esophagus. Lasers Surg Med. 2011; 43: 705-12.

6. Sunar U, Rohrbach D, Rigual N, Tracy E, Keymel K, Cooper MT, et al. Monitoring photobleaching and hemodynamic responses to HPPH-mediated photodynamic therapy of head and neck cancer: a case report. Opt Express. 2010; 18: 14969-78.

7. Bellnier DA, Greco WR, Nava H, Loewen GM, Oseroff AR, Dougherty TJ. Mild skin photosensitivity in cancer patients following injection of Photochlor (2-[1-hexyloxyethyl]-2-devinyl pyropheophorbide-a; HPPH) for photodynamic therapy. Cancer Chemother Pharmacol. 2006; 57: 40-5.

8. Payne JT, McCaw DL, Casteel SW, Frazier D, Rogers K, Tompson RV. Pharmacokinetics of pyropheophorbide-a-hexyl ether in the dog. Lasers Surg Med. 1996; 18: 406-9.

9. Lobel J, MacDonald IJ, Ciesielski MJ, Barone T, Potter WR, Pollina J, et al. 2-[1-hexyloxyethyl]-2-devinyl pyropheophorbide-a (HPPH) in a nude rat glioma model: implications for photodynamic therapy. Lasers Surg Med. 2001; 29: 397-405. 
10. Mitra S, Maugain E, Bolotine L, Guillemin F, Foster TH. Temporally and spatially heterogeneous distribution of MTHPC in a murine tumor observed by two-color confocal fluorescence imaging and spectroscopy in a whole-mount model. Photochem Photobiol. 2005; 81: 1123-30.

11. Mitra S, Foster TH. In vivo confocal fluorescence imaging of the intratumor distribution of the photosensitizer mono-L-aspartylchlorin-e6. Neoplasia. 2008; 10: 429-38.

12. Foster TH, Giesselman BR, Hu R, Kenney ME, Mitra S. Intratumor administration of the photosensitizer pc 4 affords photodynamic therapy efficacy and selectivity at short drug-light intervals. Transl Oncol. 2010; 3: $135-41$.

13. Grivennikov SI, Greten FR, Karin M. Immunity, inflammation, and cancer. Cell. 2010; 140: 883-99.

14. Castano AP, Mroz P, Hamblin MR. Photodynamic therapy and anti-tumour immunity. Nat Rev Cancer. 2006; 6: 535-45.

15. Korbelik M, Cecic I. Contribution of myeloid and lymphoid host cells to the curative outcome of mouse sarcoma treatment by photodynamic therapy. Cancer Lett. 1999; 137: 91-8.

16. de Vree WJ, Essers MC, Koster JF, Sluiter W. Role of interleukin 1 and granulocyte colony-stimulating factor in photofrin-based photodynamic therapy of rat rhabdomyosarcoma tumors. Cancer Res. 1997; 57: 2555-8.

17. de Vree WJ, Essers MC, de Bruijn HS, Star WM, Koster JF, Sluiter W. Evidence for an important role of neutrophils in the efficacy of photodynamic therapy in vivo. Cancer Res. 1996; 56: 2908-11.

18. Gollnick SO, Evans SS, Baumann H, Owczarczak B, Maier P, Vaughan L, et al. Role of cytokines in photodynamic therapy-induced local and systemic inflammation. Br J Cancer. 2003; 88: 1772-9.

19. Kousis PC, Henderson BW, Maier PG, Gollnick SO. Photodynamic therapy enhancement of antitumor immunity is regulated by neutrophils. Cancer Res. 2007; 67: 10501-10.

20. Bigelow CE, Conover DL, Foster TH. Confocal fluorescence spectroscopy and anisotropy imaging system. Opt Lett. 2003; 28: 695-7.

21. Manders EMM, Verbeek FJ, Aten JA. Measurement of Colocalization of Objects in Dual-Color Confocal Images. J Microsc-Oxford. 1993; 169: 375-82.

22. Mitra S, Haidaris CG, Snell SB, Giesselman BR, Hupcher SM, Foster TH. Effective photosensitization and selectivity In Vivo of Candida Albicans by meso-tetra (N-methyl-4-pyridyl) porphine tetra tosylate. Lasers Surg Med. 2011; 43: 324-32.

23. Sun J, Cecic I, Parkins CS, Korbelik M. Neutrophils as inflammatory and immune effectors in photodynamic therapy-treated mouse SCCVII tumours. Photochem Photobiol Sci. 2002; 1: 690-5.

24. Henderson BW, Gollnick SO, Snyder JW, Busch TM, Kousis PC, Cheney $\mathrm{RT}$, et al. Choice of oxygen-conserving treatment regimen determines the inflammatory response and outcome of photodynamic therapy of tumors. Cancer Res. 2004; 64: 2120-6.

25. Nathan C. Neutrophils and immunity: challenges and opportunities. Nat Rev Immunol. 2006; 6: 173-82.

26. DiStasi MR, Ley K. Opening the flood-gates: how neutrophil-endothelial interactions regulate permeability. Trends Immunol. 2009; 30: 547-56.

27. Chen B, Pogue BW, Luna JM, Hardman RL, Hoopes PJ, Hasan T. Tumor vascular permeabilization by vascular-targeting photosensitization: effects, mechanism, and therapeutic implications. Clin Cancer Res. 2006; 12: 917-23.

28. Mantovani A, Cassatella MA, Costantini C, Jaillon S. Neutrophils in the activation and regulation of innate and adaptive immunity. Nat Rev Immunol. 2011; 11: 519-31.

29. Fleming TJ, Fleming ML, Malek TR. Selective expression of Ly-6G on myeloid lineage cells in mouse bone marrow. RB6-8C5 mAb to granulocyte-differentiation antigen (Gr-1) detects members of the Ly-6 family. J Immunol. 1993; 151: 2399-408.

30. Nakano H, Yanagita M, Gunn MD. CD11c(+)B220(+)Gr-1(+) cells in mouse lymph nodes and spleen display characteristics of plasmacytoid dendritic cells. J Exp Med. 2001; 194: 1171-8.

31. Rose S, Misharin A, Perlman H. A novel Ly6C/Ly6G-based strategy to analyze the mouse splenic myeloid compartment. Cytometry A. 2012; 81: 343-50.

32. Cummings RJ, Mitra S, Lord EM, Foster TH. Antibody-labeled fluorescence imaging of dendritic cell populations in vivo. J Biomed Opt. 2008; 13: 044041.

33. Abi Abdallah DS, Egan CE, Butcher BA, Denkers EY. Mouse neutrophils are professional antigen-presenting cells programmed to instruct Th1 and Th17 T-cell differentiation. Int Immunol. 2011; 23: 317-26.

34. Iking-Konert C, Cseko C, Wagner C, Stegmaier S, Andrassy K, Hansch GM. Transdifferentiation of polymorphonuclear neutrophils: acquisition of CD83 and other functional characteristics of dendritic cells. J Mol Med (Berl). 2001; 79: 464-74. 